Saudi Journal of Biomedical Research

Abbreviated Key Title: Saudi J Biomed Res ISSN 2518-3214 (Print) |ISSN 2518-3222 (Online)

\title{
Treatment of Ocular Hypertension: When to Treat as Decided by the Practitioner in Comparison to a Risk Calculator
}

Dr. Shyam Kumar Gupta ${ }^{1}$, Dr. Bhaskar Agarwal ${ }^{2 *}$

${ }^{1}$ (MBBS, MS) Singapore Eye Research Institute, Singapore

${ }^{2}$ Associate Professor, Department of Prosthodontics, King George's Medical University, Shah Mina Rd, Chowk, Lucknow, Uttar Pradesh 226003, India

DOI: $10.36348 /$ sjbr.2021.v06i02.002

| Received: 20.01.2021 | Accepted: 03.02.2021 | Published: 06.02.2021

*Corresponding author: Dr. Bhaskar Agarwa

\section{Abstract}

Aims: To compare the decision to treat between the practitioner and Star and Star II calculator for ocular hypertension patients. Study Design: A comparative cross sectional retrospective analytical study. Method: A retrospective analytical study, conducted at Glaucoma clinic of Dr. R M L hospital. All files of diagnosed cases of ocular hypertension were considered for the study. Information was gathered for each of the following 6 risk factors- Age, Sex, Intra-Ocular pressure, Vertical Cup-Disc ratio, Pattern Standard Deviation (PSD), Central Corneal Thickness (CCT). Results were compared with the clinical decision for treatment taken by glaucoma practitioners well versed with OHTS and EGPS guidelines for treatment of OHT and having more than ten years of experience in their field. Result: Records of a total of 1780 files registered in the glaucoma clinic of the hospital were screened. 202 files were cases of OHT. After applying the strict STAR II inclusion criteria only 32 patient files were selected for the study. These results were subjected to statistical analysis using Mcnemar test which revealed that there was no statistical difference between the two $(\mathrm{p}=$ $0.3018)$ at $95 \%$ confidence interval. Conclusion: Our study found no statistically significant difference between the decision to treat by the two modalities i.e. STAR II (using 15\% cut-off) and the clinician decision. STAR calculator can be of help in management of a case of OHT.

Keywords: Ocular Hypertension, Intra-Ocular pressure, Vertical Cup-Disc ratio, Pattern Standard Deviation (PSD), Central Corneal Thickness (CCT).

Copyright $\odot 2021$ The Author(s): This is an open-access article distributed under the terms of the Creative Commons Attribution 4.0 International License (CC BY-NC 4.0) which permits unrestricted use, distribution, and reproduction in any medium for non-commercial use provided the original author and source are credited.

\section{INTRODUCTION}

An eye specialist is often faced with a dilemma when treating a case of ocular hypertension $(\mathrm{OHT})$. These patients with high pressures are at the moderate risk of developing primary open angle glaucoma. Even in OHTS it was seen that the observation group was statistically more likely to develop glaucoma as compared to the treatment group. The timing to start treatment becomes a very crucial decision for the ophthalmologist [1-3].

The risk-benefit ratio needs to be calculated in each case before starting a patient on anti glaucoma treatment. A rational choice has to be made whether the clinical use of drugs is essential or not for the reduction of Intra Ocular Pressure in patients of ocular hypertension. The first validated, predictive model for glaucoma risk assessment was developed in 2005 at the Hamilton Glaucoma Centre, University of California. This was the basis for STAR It was derived from published results of OHTS and then validated in an independent population as part of the National Eye Institute-sponsored Diagnostic Innovations in Glaucoma Study (DIGS) at the university of California, San Diego. The STAR II calculator is a 5-year glaucoma risk assessment tool based on a pooled, validated, predictive model that evolves the data and calculations derived from STAR obtained independently in the Ocular Hypertension Treatment Study (OHTS) and European Glaucoma Prevention Study (EGPS) [5-8].

The original STAR risk calculator used the risk factors enumerated by OHTS: age, vertical cup-todisc ratio, Humphrey visual field pattern standard deviation, IOP, CCT, and presence or absence of diabetes (OHTS found that the presence of diabetes is protective against glaucoma). The STAR calculator and the Devers calculator both account for this finding [2, 3, 5]. The newer OHTS-EGPS calculator (STAR II) considers similar risk factors, but excludes diabetes 
from the calculation of risk.It calculates the risk in percentage and recommends treatment if the risk percentage is more than $15 \%$ [6-10].

The clinician on the other hand uses his own experience and skills to decide whether treatment is required in cases of OHT. As per authors knowledge there is no retrospective analytical study available to compare the utility of this tool to glaucoma experts real decisions which has already being taken. So we conducted this study to know whether we can confidently rely on STAR decision or not.

\section{MATERIALS AND METHOD}

A retrospective analytical study, conducted at Glaucoma clinic of Dr. R M L hospital. Records of the all registered cases in the glaucoma clinic of the hospital were screened. All files of diagnosed cases of ocular hypertension were considered for the study. These files were further scrutinized and only those files which were able to fulfill the strict criteria for the star II calculator were included in the study.

\section{Inclusion Criteria}

1) Average of the untreated IOP of both eyes determined over 2 to 4 visits within 6 months.

2) Average of 3 different measurements of CCT from both eyes at the same visit.

3) Average of PSD from 2 visual fields from both eyes.

4) Average of vertical cup disc ratio by contour of both eyes.

\section{Exclusion Criteria}

1) Presence of any other intra ocular pathology or surgery that might lead to erroneous disc or HVF interpretation.

2) Refractive procedure of the cornea

3) History of using any AGT in the past 6 months

All patients underwent following baseline and follow-up ocular examination including: Medical and ocular history (especially Diabetes mellitus), Refractive and best corrected visual acuity, Detailed slit lamp examination, Goldman applanation tonometery, Gonioscopy, Ditated optic nerve head examination with plus 78D plus $90 \mathrm{D}$, Sterioscopic optic disc photography, Visual field examination (Humphreys 242 test) and Central Corneal Thickness.

Patients information was gathered for each of the following 6 risk factors: Age, Sex, Intra-Ocular pressure, Vertical Cup-Disc ratio, Pattern Standard Deviation (PSD), Central Corneal Thickness (CCT). Five year risk of conversion to open angle glaucoma was calculated using the scoring tool in percentage. It divided the risk into three categories and recommended actions for the same.
Table-1: Recommended actions of glaucoma calculator

\begin{tabular}{|l|l|}
\hline Level of risk over 5 years & Recommended actions \\
\hline Low $(<5 \%)$ & Observe and monitor \\
\hline Moderate $(5 \%$ to $15 \%)$ & Consider treatment \\
\hline High $(>15 \%)$ & Treatment \\
\hline
\end{tabular}

The results from the STAR II calculator were then compared with the clinical decision for treatment taken by glaucoma practitioners well versed with OHTS and EGPS guidelines for treatment of OHT and having more than ten years of experience in their field.

Statistical Analysis: These results were subjected to statistical analysis using Mcnemar test.

\section{RESULTS}

Records of a total of 1780 files registered in the glaucoma clinic of the hospital were screened. 202 files were cases of OHT. After applying the strict STAR II inclusion criteria only 32 patient files were selected for the study. Out of which clinician has decided 17 OHT patients not to treat (NT) and 15 OHT patients to take treatment (TT), where as Star Calculator suggested 22 OHT patients in NT group and $10 \mathrm{OHT}$ patients in TT group.

Table-2: Correlation between clinician and glaucoma calculator

\begin{tabular}{|l|l|l|l|}
\hline Star Calculator & \multicolumn{3}{|l|}{ Clinician } \\
\hline & NT & TT & Total \\
\hline NT & 12 & 10 & 22 \\
\hline TT & 5 & 5 & 10 \\
\hline Total & 17 & 15 & 32 \\
\hline
\end{tabular}

NT: Not to treat, TT: Treat

These results were subjected to statistical analysis using Mcnemar test which revealed that there was no statistical difference between the two ( $\mathrm{p}=$ 0.3018 ) at $95 \%$ confidence interval.

\section{DISCUSSION}

The STAR calculator can be a bridge between a general practitioner and the glaucoma specialist [1]. An experienced general practitioner but lacking enough glaucoma knowledge can always rely on it for crucial decision to start treatment in OHT [5].

Our study found no statistically significant difference between the decision to treat by the two modalities i.e. STAR II (using 15\% cut-off) and the clinician decision. STAR calculator can be of help in management of a case of OHT $[2,3,5,7]$. Though, It is important to remember that this calculation only applies to patients that have not been diagnosed with glaucoma and have never been treated. Also, the strict guidelines of the calculator should be adhered to while using the calculator. It should also be stressed that the calculator 
should be an important accessory tool to decide initiation of treatment and not the sole criteria for it.

\section{CONCLUSION}

Our study found no statistically significant difference between the decision to treat by the two modalities i.e. STAR II (using $15 \%$ cut-off) and the clinician decision. STAR calculator can be of help in management of a case of OHT and STAR calculator for glaucoma treatment can be safely used by general ophthalmologist.

\section{REFERENCES}

1. Mansberger, S. L. (2004). A risk calculator to determine the probability of glaucoma. Journal of glaucoma, 13(4), 345-347.

2. Medeiros, F. A., Weinreb, R. N., Sample, P. A., Gomi, C. F., Bowd, C., Crowston, J. G., \& Zangwill, L. M. (2005). Validation of a predictive model to estimate the risk of conversion from ocular hypertension to glaucoma. Archives of ophthalmology, 123(10), 1351-1360.

3. Ocular Hypertension Treatment Study Group, \& European Glaucoma Prevention Study Group. (2007). Validated prediction model for the development of primary open-angle glaucoma in individuals with ocular hypertension. Ophthalmology, 114(1), 10-19.

4. Wilson, P. W., Castelli, W. P., \& Kannel, W. B. (1987). Coronary risk prediction in adults (the Framingham Heart Study). The American journal of cardiology, 59(14), G91-G94.
5. Gordon, M. O., Beiser, J. A., Brandt, J. D., Heuer, D. K., Higginbotham, E. J., Johnson, C. A., ... \& Ocular Hypertension Treatment Study Group. (2002). The Ocular Hypertension Treatment Study: baseline factors that predict the onset of primary open-angle glaucoma. Archives of ophthalmology, 120(6), 714-720.

6. Brindle, P., Beswick, A., Fahey, T., \& Ebrahim, S. (2006). Accuracy and impact of risk assessment in the primary prevention of cardiovascular disease: a systematic review. Heart, 92(12), 1752-1759.

7. Mansberger, S. L., \& Cioffi, G. A. (2006). The probability of glaucoma from ocular hypertension determined by ophthalmologists in comparison to a risk calculator. Journal of glaucoma, 15(5), 426431.

8. Bobbio, M., Fubini, A., Detrano, R., Shandling, A. H., Ellestad, M. H., Clark, J., ... \& Martinez-Caro, D. (1994). Diagnostic accuracy of predicting coronary artery disease related to patients' characteristics. Journal of clinical epidemiology, 47(4), 389-395.

9. Poses, R. M., Cebul, R. D., \& Wigton, R. S. (1995). You can lead a horse to water-improving physicians' knowledge of probabilities may not affect their decisions. Medical Decision Making, 15(1), 65-75.

10. Haas, J. S., Kaplan, C. P., Gregorich, S. E., PérezStable, E. J., \& Des Jarlais, G. (2004). Do physicians tailor their recommendations for breast cancer risk reduction based on patient's risk?. Journal of general internal medicine, 19(4), 302-309. 